\title{
Kaposi's Sarcoma and Lymphoedema in a HIV Sero-positive Patient: A Case Report and Literature Review
}

\author{
M. S. Turton, I. Reddy, and L. P. Turton
}

\section{ABSTRACT}

Kaposi sarcoma (KS) is one of the most common neoplasms diagnosed in HIV- seropositive subjects. $\mathrm{KS}$ is an angioproliferative disorder of endothelial origin and is a locally aggressive multicentric mucocutaneous malignant neoplasm. Aggressive HIV-KS is associated with increased HHV8 viral load, disseminated lesions, and intraoral exophytic lesions, and may also present with facial lymphoedema which may be life-threatening or mild.

\begin{abstract}
A 26 -year-old, male, presented with severe oral pain and facial swelling. He had multiple oral lesions and an incisional biopsy confirmed Kaposi sarcoma. A multiple site incisional biopsy and immunohistochemistry for HHV8 was done and based on the clinical, histopathological, and immunohistochemical results a diagnosis of HIV associated oral and cutaneous KS with severe lymphoedema was confirmed.

This aim of this case presentation is to describe a case of immunodeficiency virus-related lymphadenitis, oral and cutaneous KS associated with the complication severe lymphoedema in a HIV infected patient who was not on HAART therapy for three years after being diagnosed with HIV.
\end{abstract}

Keywords: HIV, Kaposi's sarcoma, lymphoedema, human herpes virus.
Published Online: October 26, 2021

ISSN: $2684-4443$

DOI : $10.24018 /$ ejdent.2021.2.5.96

\section{S. Turton*}

Wits University, South Africa.

(e-mail: mervyn.turton@ ${ }^{\circledR}$ wits.ac.za)

I. Reddy

JDJ Laboratory, South Africa

(e-mail: reddy.isai@gmail.com)

L. P. Turton

Turton Dental Surgery and Laboratory, South Africa.

(e-mail: lionel.turton@ ${ }^{@ m a i l . a c . z a) ~}$

*Corresponding Author

\section{INTRODUCTION}

Kaposi's sarcoma (KS) is a multicentric [1], [2], mucocutaneous, malignant neoplasm and is one of the most common locally aggressive neoplasm diagnosed in HIVseropositive subjects [1], [3]. $\mathrm{KS}$ is considered an angioproliferative disorder of endothelial origin and may affect mucosal sites, lymph nodes, and visceral organs [1], [2].

There are four clinico-epidemiological variants of KS the Mediterranean or classic KS occurs among elderly men of East European and Ashkenazic Jewish origin [4]-[6]; the epidemic of human immunodeficiency virus-associated (HIV-KS); the iatrogenic or post-transplant is associated with patients on immunosuppressive therapy KS, and the African or endemic KS which occurs in adults, adolescents, and children, with a high frequency of extracutaneous manifestations [4]-[8]. All variants of KS are considered to be caused by the human herpes virus (HHV-8) also referred to as KS-associated herpes virus (KSHV) [6]-[9] in combination with other cofactors such as severe immune suppression, angiogenic mediators, or genetic predisposition, which all appear to be necessary for the development of KS [4], [6], [9], [10]. HIV-KS is common in African countries where there are widespread HIV infections, an endemic HHV8 infection, and where there is poor availability of antiretroviral medication [12]-[14].
Oral Kaposi's sarcoma (OKS) commonly occurring tumour with lesions that may be single or multifocal, non-pigmented to brownish-red or violaceous in colour [15]-[17], on the hard and soft palate, gingiva, and dorsal tongue [6], [17]-[19]. OKS may be associated with pain, bleeding, and functional interferences [17]-[19]. The initial lesions may manifest initially as ecchymotic or erythematous patches [20]-[22] which develop into nodular, that progress to papular, and ultimately become confluent forming large exophytic forms which could invade bone and create tooth mobility and morbidity [14], [23], [24].

Oral HIV-KS is frequent and is associated with a poor prognosis and has a higher mortality rate than in patients with only cutaneous HIV-KS and patients with oral HIV-KS have a 24-month median survival rate compared to only cutaneous HIV- KS that has a 72-month median survival rate [3], [21], [22].

HIV-KS may develop at any stage of HIV infection including the stage of early HIV-seropositivity, but it is more prevalent at a lower CD4+ T-cell count [21], [22].

Aggressive HIV-KS is associated with increased HHV8 viral load, disseminated lesions, and intraoral exophytic lesions, and may also present with facial lymphoedema which may be life-threatening or mild [22], [23].

In some cases, HIV-KS may manifest as an immune reconstitution inflammatory syndrome (IRIS) which is consequent with the improvement of the host immune status, 
after the patient is administered antiretroviral therapy [21].

The initial presentation of HIV-KS is usually in the mouth in an estimated $22 \%$ of HIV-seropositive subjects with $\mathrm{KS}$, and about $71 \%$ of subjects with HIV-KS will at some stage present with OKS [25]-[27].

\section{AIM}

The aim of this case presentation is to describe a case of immunodeficiency virus-related lymphadenitis, oral and cutaneous KS associated with the complication severe lymphoedema in a HIV infected patient who was not on HAART therapy for three years after being diagnosed with HIV.

\section{CASE PRESENTATION}

A 26 years old, black male complained of severe oral pain and bleeding gums which impeded his ability to eat and his ability to take medication. The patient also complained that his facial swelling was affecting his ability to open his eyes adequately enough thus impairing his vision. The patient had been attended to at a provincial hospital where a cervical lymph node measuring $15 \times 10 \times 4 \mathrm{~mm}$ was excised.

\section{A. Clinical Evaluation}

The patient presented with severe lymphoedema which was pronounced around his eyes (Fig. 1).

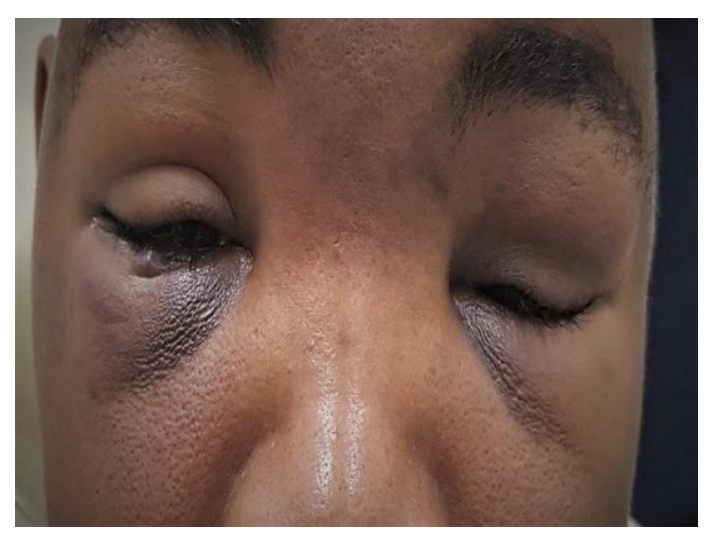

Fig. 1. Face of patient with severe lymphoedema.

An intraoral examination found poor oral hygiene with moderate dental plaque and calculus accumulation; gingival bleeding was generalized. There was no radiographic evidence of bone loss, however, the hard and soft palate had Candida infection. A generalized brownish to red and redpurplish oral lesions that were multifocal in distribution, on the gingiva, hard palate, and soft palate were noted. Some of the lesions were papular other lesions were ulcerated soft nodular lesions, additionally, several telangiectasias were seen on the surface of the lesions. The red to brownish soft outgrowths were particularly pronounced on the anterior buccal gingiva, at the 14 to 24 regions, which appeared to be spreading to the lip region (Fig. 2).

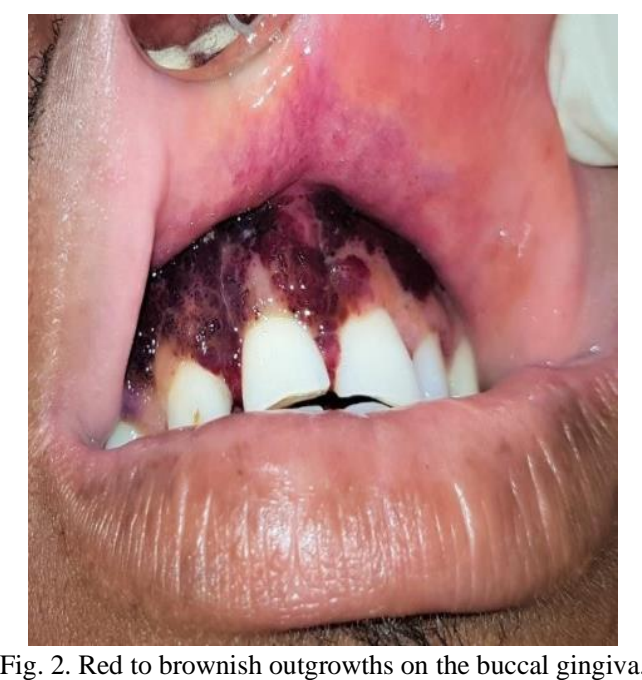

On the palate of the 16,11 , and 21,26 , and 27 regions a red purplish, ulcerated, soft nodular/papular lesions were present and further clinical examination also revealed the presence of Candida infection on the hard palate and soft palate (Fig. 3).

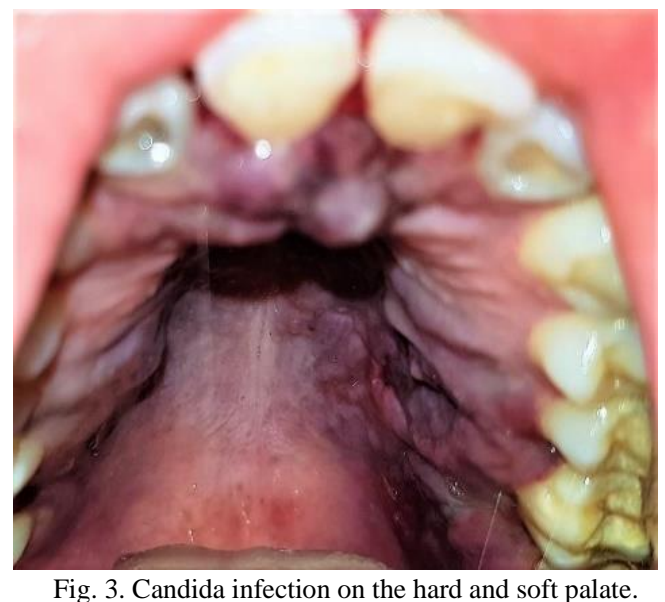

The patient had tested positive for HIV and his CD4+ Tcell count at the time of HIV-KS diagnosis was 107 cells $/ \mathrm{mm}^{3}$. He had tested positive for Tuberculosis and had a persistent cough, clubbing. Tender and mobile palpable lymphadenopathy was present on the right and left submandibular and posterior cervical chains.

\section{B. Histology}

\section{1) Incisional biopsy 28 region of palate}

This tissue section displays mild acanthosis of the squamous mucosa with an underlying spindle cell proliferation. The tumour is present at the deep margin and is composed of tight fascicles of spindled-shaped cells with mildly pleomorphic, vesicular nuclei. The spindled cells surround vascular spaces of varying caliber, including prominent slit-like vascular spaces with scattered mitotic figures. Extravasated red blood cells and mixed inflammatory cells, including scattered plasma cells and haemosiderin deposition can be noted (Fig. 4). 


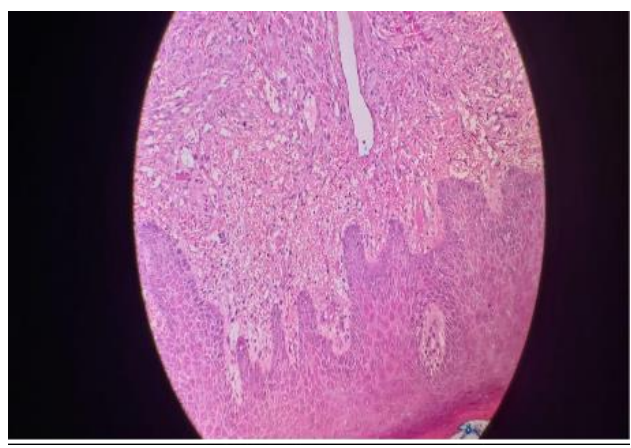

Fig. 4. Extravasated red blood cells with haemosiderin deposition.

Florid Superficial Candida organisms are seen on the surface of the Stratum Corneum. The tumour is present at the lateral and deep margins. Features are those of a spindle cell tumour consistent with Kaposi's sarcoma, which is present at the Lateral and Deep Margins of the Specimen. Ulceration and mixed inflammation are seen with an area suggestive of an abscess at the deep margin with florid superficial Candida infection (Fig. 5).

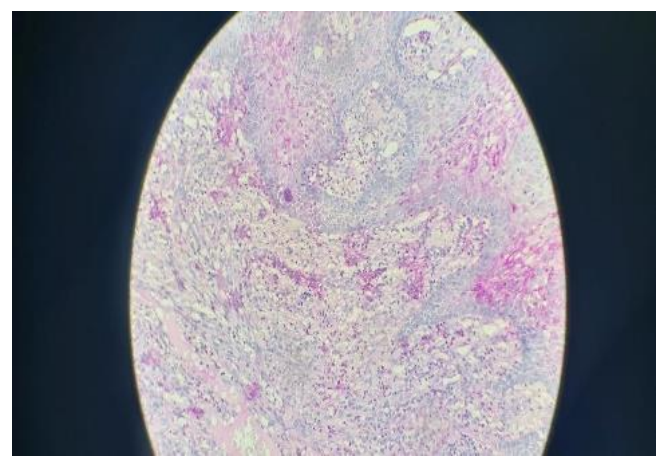

Fig. 5. Ulceration and mixed inflammation with florid superficial Candida infection.

\section{Excisional Biopsy of Cervical Lymph Gland}

Histologically it demonstrated a think capsule with a vasoformative spindle cell tumour-forming slit-like and larger dilated vascular channels. Red blood cell extravasation and surrounding plasma cells are observed. Reactive Lymphoid follicles, follicle lysis, interfollicular plasmacytosis, and proliferation of small blood vessels were observed. The tumour cells expressed HHV-8 (Fig. 6).

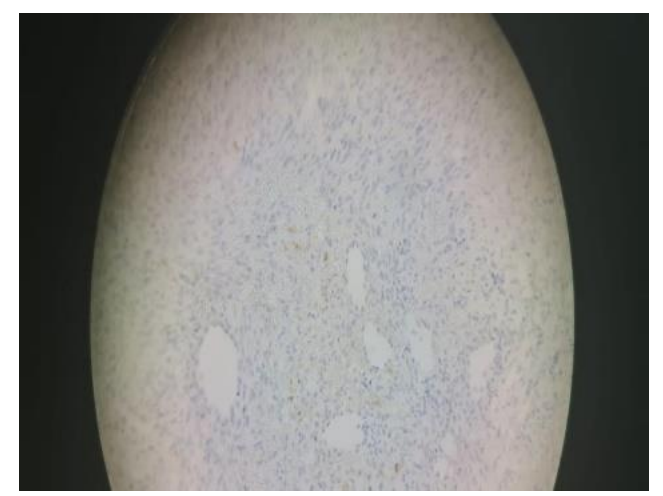

Fig. 6. HHV-8 expressed in tumour cells.

\section{Diagnosis}

Typical KS lesions do not exhibit marked cellular pleomorphism, necrosis, or many mitotic figures, though, mildly atypical endothelial cells and monomorphic spindle cells may be observed [20], [21], [23]. Therefore, it is also possible that a biopsy can be initially misdiagnosed and for that reason, KS should be diagnosed with histology and immunohistochemistry due to a large range of similarities within diseases [17], [20], [38]. A multiple site incisional biopsy and immunohistochemistry for HHV8 was done and based on the clinical, histopathological, and immunohistochemical results a definitive diagnosis of HIV associated oral and cutaneous Kaposi's sarcoma and human immunodeficiency virus-related lymphadenitis with severe lymphoedema was confirmed.

\section{DISCUSSION AND LITERATURE REVIEW}

$\mathrm{KS}$, a multifocal neoplasm of vascular endothelial cell origin that was first described by the Hungarian dermatologist Moritz Kaposi in 1872 [38], [39].

The aetiology of $\mathrm{KS}$ is unknown. However, many epidemiological and environmental factors play a role in the development of the disease.

In the last decade, evidence has suggested that infection with a recently discovered herpes virus, namely KS herpes virus or $\mathrm{HHV}-8$, is responsible for all forms of KS [8].

Before the HIV/AIDS epidemic, KS was rarely seen and occurred mainly on elderly men of Jewish, Italian, and Slavic ancestry [4]-[6]. However, as HIV/ AIDS became a widespread disease, KS became one of the most common malignancies associated with HIV/AIDS infection [6], [7] and the presence of $\mathrm{KS}$ lesions in HIV-seropositive is indicative subjects of AIDS [5], [7], [8].

The oncovirus human herpesvirus 8 (HHV-8) referred to as the $\mathrm{KS}$-associated herpes virus (KSHV) is the causative organism in approximately $90 \%$ of $\mathrm{KS}$ cases implicating HHV-8 interleukin-6 (IL-6), which initiates a mitogenic effect [6], [7], [28]. In immunocompetent individuals, HHV8 is asymptomatic, however, in immunocompromised hosts such as in HIV sero-positive patients it is the causative organism in the development of neoplastic disorders, such as KS [2], [4], [23]. KSHV can infect oral epithelial cells and it remains in the oral cavity of healthy individuals even in the presence of a healthy immune system which may control the persistent infection to keep it subclinical for the lifetime of the host [8], [28], 29].

\section{A. The Course of the Disease}

OKS lesions initially manifest as a proliferation of small veins and capillaries around one or more dilated vessels may be associated with bleeding, pain, and functional interferences caused by the tumour [23], [28], [29]. OKS lesions are mostly observed in young adult patients, often affecting mucous, skin, and internal organs [23], [28], [29].

It has an aggressive clinical course [4], [28], [29]. KS is reported to be the most prevalent type of cancer in HIV+ patients that have not received treatment, and it is associated with the infection by HHV-8 [29]-[31].

The pace of HIV- KS is quite variable with a very poor prognosis, though this is strongly influenced by the immune status of the patient ranging from a slow progression requiring none to basic therapy or aggressively progressive disease [7], [30], [31]. 
OKS lesion is initially a flat brown or reddish-purple macule that does not blanch with pressure and generally begins as multiple lesions or as a single lesion of the oral mucosa [29], [32]. The lesion may develop into plaques or nodules, and further disseminating to lymph nodes and organ systems as the malignancy progresses and tends to be spread, multiply in number, become more nodular or coalesce in association with immunological deterioration and the drop in the count of CD4 cells [27], [29], [32].

As the lesion progresses it eventually reaches the nerve structure which elicits pain, which was the case in this report as the patient complained about pain and had some spontaneous bleeding, and difficulty in eating [6], [28].

\section{DIFFERENTIAL DiAgNOSIS}

The differential diagnosis of KS includes and for early lesions can be included lesions such as benign vascular proliferation like hemangioma, bacillary angiomatosis, and pyogenic granuloma [18], [29], [30], [32].

Clinically KS must be differentiated from benign and malignant tumours and early lesions must exclude vascular lesions such as benign lymphangioendothelioma, hemangiomas, acroangiodermatitis ecchymosis arteriovenous hemangiomas (at the papular or nodular stage), and also lymphangiomas or deeply located low-grade mucoepidermoid carcinoma (at initial stages) [6], [29], [31].

Lesions such as bacillary angiomatosis can appear as multifocal KS lesions though this is rare. KS may resemble pyogenic granuloma or peripheral giant cell proliferation when it occurs on the alveolar ridge [17], [18], [32].

Cellular and more advanced lesions must be differentiated microscopically KS from angiosarcoma, Kaposiform hemangioendothelioma lymphoma, spindle cell haemangioendothelioma, aneurysmal fibrous histiocytoma, spindle cell hemangioma, fibrosarcoma (advanced KS lesion), dermatofibrosarcoma protuberans, vascular or pilar leiomyomas, well-differentiated angiosarcoma (early KS lesion) and even undifferentiated squamous cells carcinoma arteriovenous malformations [6], [15], [23], [32].

An accurate clinical history and cautious histological sampling are of paramount importance for establishing the diagnosis of KS and thus distinguishing it from other lesions. It has been suggested that $\mathrm{KS}$ as a prognostic marker in nontreated HIV+ patients [26], [29], [30] with a worse prognosis predicted for HIV-positive patients afflicted with Kaposi's sarcoma however other studies refute this suggestion [15], [25], [29]. In the discussed case, by either ignoring the fact of being an HIV bearer or not presenting for treatment, the patient developed cutaneous and oral KS and severe lymphoedema due to the lack of treatment for the infection [29], [30], [33].

\section{TREATMENT}

Some studies found that patients with OKS had a higher mortality rate than those with solely cutaneous KS lesions and they further reported that patients with a CD4 cell count of at least $150 / \mu \mathrm{L}$ and $\mathrm{OKS}$ had a similar mortality risk as patients with a CD4 cell concentration of less than $150 / \mu \mathrm{L}$. and cutaneous KS [3], [4], [6], [33].

Other studies suggest that the emergence of KS in HIV+ patients is associated with CD4 T-lymphocyte counts below $200 \mathrm{cell} / \mu \mathrm{l}$ [5], [6], [15].

The literature reports that KS first appears in the oral cavity in $22 \%$ of $\mathrm{HIV}+$ patients, and up to $71 \%$ of $\mathrm{HIV}$-infected patients develop oral KS together with skin and visceral involvement [5], [28], [34]. Similarly, in this reported case showed oral lesions associated with a skin nodular lesion that arose after the emergence of oral lesions. In the reported case, the presence of extensive oral lesions raised suspicions of KS associated with the HIV infection [5], [19], [29], [31], [32].

The treatment selection for KS includes local treatments such as surgical excision or doses of radiotherapy for small local lesions [23], [28], [29]. Disseminated and symptomatic forms of KS are treated with systemic treatment and staging of the tumour based on the clinical signs is indicated prior to treatment. Systemic treatments include chemotherapy or polychemotherapy with adriamycin, vinblastine and bleomycin; and/or biological therapy particularly with recombinant interferon- $\alpha$ due to its anti-angiogenetic and immunomodulating effects [4], [6], [14], [23].

These treatment modalities include HAART, local surgical excision, electrosurgery, chemotherapy, radiation therapy, cryotherapy, and laser therapy and they may be isolated or in combination. Further treatment options can be topical therapy in form of nitrogen mustard or imiquamod, alpha- and betainterferon, photodynamic therapy, and intralesional or systemic injections with cytostatic agents [5], [29], [36].

Small and single KS lesions can be surgically excised [19] and local recurrence is rare after complete excision of the primary lesion. However, in the event of relapse, it occurs six months to two years after the procedure [19], [29].

Systemic therapy is indicated in the cases there is a failure to respond to local therapy, extensive Kaposi sarcomaassociated with lymphoedema, visceral involvement, extensive and rapidly progressing Kaposi sarcoma [6], [29].

It has been reported that the administration of nonnucleotide reverse transcriptase inhibitor (NNRTI)-based therapy decreased the incidence of KS AND PI- and NNRTIbased HAART regimens are equally protective against KS [6], [18], [29].

The literature suggests that new cases of KS commonly occur in patients with advanced immunosuppression and are not on antiretroviral therapy, which is indicative of delayed diagnosis and treatment of HIV-1 infection [18], [29], [34].

Current evidence suggests that $\mathrm{KS}$ often goes into remission with HAART, and HIV-infected patients on antiretroviral therapy are inflicted with a less aggressive form of KS than those who are not on antiretroviral therapy at the time of diagnosis ${ }^{[19,29]}$.

The patient in the case was not undergoing HAART therapy and hence he presented with the dissemination of oral and skin lesions associated with severe immunosuppression as his CD count was 107 cells/mm3 [19], [27], [29].

$\mathrm{KS}$ is considered a progressive malignancy that may disseminate extensively into various organ systems and lymph nodes. KS treatment modalities have been significantly improving with a variable prognosis depending on the form of the KS and the patient's immune status [3], [18], [29] hence the specific needs of the patient must be 
carefully considered before deciding on a specific treatment plan. It has been established that effective HAART therapy causes regression of established lesions and decreases the incidence of KS in persons infected with HIV [18], [35]. There is uncertainty about the mechanism of action that produces an improvement in the clinical course of HIV-KS whether it is caused by a direct antiviral effect against HHV8 or can be attributed to improvement in the host-immune status response [18], [29], [32].

The pattern of KS incidence has been significantly reduced by the introduction of HAART therapy which targets the underlying HIV infection, thereby improving immune function and, as far as we know, leading to improvements in existing KS [15], [18], [35].

Recent treatment modalities are focused on HHV-8 or at the inflammatory cytokine or angiogenic milieu necessary for KS growth [28], [29].

Systemic chemotherapy is administered for extensive visceral involvement, for which there is no other effective treatment, however, extensive systemic therapy can result in the patient's increased susceptibility to infections and other neoplasms due to further depression of the immune system [6], [18], [32].

Currently, there is no permanent cure for HIV-AIDS however most patients with HIV -KS succumb to other complications such as opportunistic infections [34], [18], [29]. The patient was treated with highly active antiretroviral therapy (HAART) at the time of presenting with KS as well as additional chemotherapy after the confirmation of the diagnosis KS with oral and lymphoedema and will be periodically monitored.

\section{CONCLUSION}

Effective HAART reduces HIV load with subsequent improvement in the host- immune status, though does not directly influence HHV8 replication, it improves the clinical manifestation and even though it does not ensure that HIV$\mathrm{KS}$ will not develop, it reduces the incidence and prevalence of HIV-KS [3], [18], [29].

The introduction of HAART late exposes people living with HIV to morbidities like KS and increased risk of early mortality.

This article reports an unusual presentation of cutaneous and oral KS lesions with the complication of severe lymphoedema in an HIV infected patient who was not on HAART therapy for three years after diagnosis. The delayed antiretroviral therapy, in the present case contributed to the presence of cutaneous and oral KS and severe lymphoedema.

\section{ACKNOWLEDGEMENTS}

We gratefully acknowledge the patient and his family for giving the necessary permission to report this case. We also gratefully acknowledge Lugelo Makhatini, Nonkuleko Makhatini, and Nosipho Ndlela for their invaluable contribution in assisting with the management of the case.

\section{AUTHOR's CONTRIBUTIONS}

Involved in conception, design, research, and manuscript writing of the study: Turton M: $70 \%$.

Contributed to interpretation and write up of the histology: Reddy I: $20 \%$.

Contributed to research and collating the information: Turton L: $10 \%$.

\section{CONFLICT OF INTEREST}

All authors declared that there was no conflict of interest.

\section{ETHICS APPROVAL AND CONSENT TO PARTICIPATE}

Informed consent has been obtained and this report was processed according to the principles expressed in the Declaration of Helsinki.

\section{REFERENCES}

[1] R. Yoshida, H. Nakayama, N. Takahashi, et al., "A case of AIDSassociated oral Kaposi's sarcoma of the tongue," J Oral MaxillofacSurg Med Pathol, vol. 26, pp. 170-4, 2014.

[2] B. Aguilar, Y. Hong, "The origin of Kaposi sarcoma tumour cells," in Kaposi Sarcoma: A Model of Oncogenesis, Pantanowitz L, Stebbing J, and Dezube B, Eds., 2012, pp. 123-138, Research Signpost, Kerala, India, View at: GoogleScholar.

[3] R. A. Khammissa, L. Pantanowitz, L. Feller, "Oral HIV-Associated KaposiSarcoma: A Clinical Study from the Ga-Rankuwa Area, South Africa," AIDS Res. Treat: 873171, 2012. doi:10.1155/2012/873171. Epub2012Sep12.PMID:23008762: PMID:PMC3447356.

[4] E. Ruocco, V. Ruocco, M. L. Tornesello, et al., 'Kaposi's sarcoma: etiology and pathogenesis, inducing factors, causal associations, and treatments: facts andcontroversies," Clin Dermatol., vol. 31, pp. 413 22, 2013.

[5] H. Fukumoto, T. Kanno, H. Hasegawa, H. Katano, "Pathology of Kaposi>s Sarcoma-Associated Herpesvirus Infection," Front Microbiol., Aug 25;2:175, 2011. doi:10.3389/fmicb.2011.00175.

[6] M. Fatahzadeh, M. Robert, A. Schwartz, "Oral Kaposi's sarcoma: a reviewand update," International Journal of Dermatology, vol. 52, Issue 6, pp. 666-672, 2013, 17 May 2013. https://doi.org/10.1111/j.1365-4632.2012.05758.x.

[7] L. E. Cavallin, P. Goldschmidt-Clermont, E. A. Mesri, "Molecular and cellular mechanisms of KSHV oncogenesis of Kaposi's sarcoma associated withHIV/AIDS," PLoSPathog.; 10: e1004154, 2014.

[8] E. Cesarman, B. Damania, S. E. Krown, J. Martin, M. Bower, D. Whitby, "Kaposi sarcoma, Nat Rev Dis Primers," Jan 31;5(1):9, 2019. Doi: 10.1038/S41572-019-0060-9.

[9] A. Pivovar, C. Chaiben, F. Gil, A. de Lima, "Oral Kaposi's sarcoma in immunosuppressed patients - report of cases .rsbo," (Online)., vol. 10, n. 1, pp. 89-95, 2013. ISSN 1984-5685.

[10] A. Mosam, H. Carrara, F. Shaik et al., "Increasing incidence of Kaposi's sarcoma in black South Africans in KwaZulu-Natal, South Africa (1983-2006),"International Journal of STD and AIDS, vol. 20, no. 8 pp. 553-556, 2009. View at: Publisher Site | Google Scholar.

[11] A. Mosam, J. Aboobaker, F. Shaik, "Kaposi's sarcoma in sub-Saharan Africa: a current perspective," Current Opinion in Infectious Diseases, vol. 23, no. 2, pp. 119-123, 2010. View at: Publisher Site | Google Scholar.

[12] L. Pantanowitz, R. A. Khammissa, J. Lemmer, L. Feller, "Oral HIVassociated Kaposi sarcoma," J Oral Pathol Med., vol. 42(3), pp. 201 7, 2013. doi:10.1111/j.1600-0714.2012.01180.x.

[13] L. L. Patton, "Oral lesions associated with human immunodeficiency virus disease," Dent Clin North Am.; vol. 57, pp. 673-98, 2013.

[14] N. Cloarec, O. Faucher, S. Bregigeon, et al., "Kaposi's sarcoma in a treated and well-controlled HIV infected patient: discussion on the role ofimmunosenescence," HIV \& AIDS Rev., vol. 13(4), pp. 131-4, 2014

[15] I. Zaraa, I. Labenne, N. Guellali, et al., "Kaposi's sarcoma: epidemiological, clinical, anatomopathological and therapeutic features in 75 patients," Tunis Med., vol. 90, pp. 116-21, 2012. 
[16] R. Wild, M. C. Balmer, "Have we forgotten? Oral Manifestations of Kaposi's Sarcoma," Sex Transm Infect., vol. 91(5), pp. 345, 2015. doi: 10.1136/sextrans-2015- 052116.

[17] E. Rohrmus, J. Thoma-Greber, R. Bogner, M. Röcken, "Outlook in oral and cutaneous Kaposi's sarcoma," The Lancet, vol. 356, no. 9248, p. 2160, 2000. Viewat: Publisher Site | Google Scholar.

[18] J. Schneider, D. Dittmer, "Diagnosis and treatment of Kaposi Sarcoma,"Am J Clin Dermatol., vol. 18(4), pp. 529-539, 2017. Doi: 10. 1007/s40257-017-0270-4.

[19] C. Donavan, H. Patel, S. Shetty, L. Bierman, J. M. Lustbader, A. M. Oboh-Weilke, "Aggresive conjunctival Kaposi sarcoma as the initial manifestation of acquired immunodeficiency syndrome," Am J Ophthamol Case Rep., Jul, 2020. Doi: 10.1016/j.ajoc.2020.100832. 16; 19:100832. PMID: 32715160.

[20] L. Feller, J. Lemmer, "Insights into pathogenic events of HIVassociated Kaposi sarcoma and immune reconstitution syndrome related Kaposi sarcoma," Infectious Agents and Cancer, vol. 3, article 1, 2008.View at: Publisher Site | Google Scholar.

[21] M. Bower, M. Nelson, A. Young et al., "Immune reconstitution inflammatory syndrome associated with Kaposi's sarcoma," Journal of Clinical Oncology, vol. 23, no. 22, pp. 5224-5228, 2005. View at: Publisher Site | Google Scholar.

[22] J. Epstein, R. Cabay, M. Glick, "Oral malignancies in HIV disease: changes in disease presentation, increasing understanding of molecular pathogenesis, and current management," Oral Surgery, Oral Medicine, Oral Pathology, Oral Radiology and Endodontology, vol. 100, no. 5, pp. 571-578, 2005. View at: Publisher Site |Google Scholar.

[23] A. Faden, M. AlSheddi, M. AlKindi, L. Alabdulaaly, "Oral Kaposi Sarcoma in HIV-seronegative Saudi patient: literature review and case report," Saudi Dent J., vol. 29(3), pp. 129-134, 2017.

[24] K. Shetty, "Management of oral Kaposi's sarcoma lesions on HIVpositive patient using highly active antiretroviral therapy: Case report and a review of the literature," Oral Oncology Extra, vol. 41(9), pp. 226-229, 2005. Doi :10.1016/.ooe.2005.06.006.

[25] R. Lupia, P. B. Wabuyia, P. Otiato, C. T. Fang, F. J. Tsai, "Risk factors for Kaposi's sarcoma in human immunodeficiency virus patients after initiation of antiretroviral therapy: anested case-control study in Kenya," $J$ Microbiol Immunol Infect., 2015. Available at: http://www.ejmii.com/article/S1684-1182(15)00906-8/pdf.

[26] K. Stolka, P. Ndom, J. Hemingway-Foday, et al., "Risk factors for Kaposi's sarcoma among HIV-positive individuals in a case control study in Cameroon," Cancer Epidemiol.; vol. 38, pp. 137-43, 2014.

[27] H. R. Lee, R. Amatya, J. U. Jung, "Multi-step regulation of innate immunesignaling by Kaposi's sarcoma-associated herpesvirus," Virus Res., vo. 209, pp. 39-44, 2015.

[28] B. M. Jr Bell, A. Syed, S. W. Carmack, C. A. Thomas, K. F. Layton, "Disseminated Kaposi sarcoma with osseous metastases in an HIVpositive patient," Proceedings, vol. 29(1), pp. 52-4, 2016.

[29] Carvalho, L. Lucena, M. Cristina, T. Honorato, G. Andrade, R. Freitas, "Case Report Mucocutaneous Kaposi's sarcoma in an HIV-positive patient: diagnosis and treatment," J. Bras. Patol. Med. Lab., vol. 52, no. 3, 2016. Rio de Janeiro May/June 2016. https://doi.org/10.5935/1676-2444.20160033.

[30] L. L. Patton, "Oral lesions associated with human immunodeficiency virus disease," Dent Clin North Am., vol. 57, pp. 673-98, 2013.

[31] S. Mehta, A. Garg, L. K. Gupta, A. Mittal, A. K. Khare, C. M. Kuldeep, "Kaposi's sarcoma as a presenting manifestation of HIV," Indian J Sex TransmDis., vol. 32, pp. 108-10, 2011.

[32] A. Faden, M. AlSheddi, M. AlKindi, L. Alabdulaaly, "Oral Kaposi Sarcoma in HIV-seronegative Saudi patient: literature review and case report," Saudi Dent J., vol. 29(3), pp.129-134, 2017.

[33] M. A. Gaber, O. A. Bakry, W. A. Shehata, "Isolated oral Kaposi sarcoma in an HIV-negative patient: a case report," Anal Quant Cytopathol Histopathol., vol. 35(4), pp. 237-240, 2013.

[34] P. R. L. Machado, K. J. S. Farias, J. Genre, et al., "Disseminated Kaposi's sarcoma in patients with HIV infection correlates to high serum levels of IL-10,” Viral Immunol., vol. 27, pp. 1-5, 2014. 